Neue Daten zur Therapie von Brustkrebspatientinnen mit Femara ${ }^{\circledR}$

Beim San Antonio Breast Cancer Symposium im Dezember 2006 standen wieder neue Daten zur Behandlung von postmenopausalen Frauen mit östrogenrezeptor(ER)-positivem Brustkrebs im Mittelpunkt.

In einer Studie mit 12 postmenopausalen Frauen mit ER-positivem, primärem Mammakarzinom, konnten Jürgen Geisler et al., Bergen, Norwegen, bestätigen, dass die Östrogenspiegel im Plasma und im Tumorgewebe unter einer Behandlung mit Femara ${ }^{\circledR}$ (Letrozol) stärker absinken als unter Anastrozol [1]. Welche Konsequenzen das für Wirkungsspektrum und Verträglichkeit der beiden Substanzen in der klinischen Anwendung habe, müsse in weiteren Studien geprüft werden.

Einen direkten Vergleich von Letrozol und Anastrozol im Hinblick auf Lebensqualitätsparameter, Verträglichkeit und Morbidität führten J. Michael Dixon et al. an 185 postmenopausalen Frauen mit invasivem, ER-positivem Mammakarzinom durch [2]. Die Patientinnen erhielten randomisiert im Crossover-Design zuerst 12 Wochen lang Letrozol 2,5 mg/d, gefolgt von 12 Wochen Anastrozol $1 \mathrm{mg} / \mathrm{d}$ bzw. vice versa. Auch in dieser Studie waren die Estradiolwerte nach Letrozol-Behandlung niedriger als nach Anastrozol. Jeweils etwa ein Drittel aller Patientinnen gaben an, Letrozol, Anastrozol oder keinem der beiden Medikamente den Vorzug zu geben. Spektrum und Häufigkeit der Nebenwirkungen der beiden Aromatasehemmer waren vergleichbar. 19 Patientinnen brachen ihre Teilnahme an der Studie ab, davon 4 noch bevor sie die erste Studienmedikation erhalten hatten. 8 Patientinnen der Letrozolgruppe und 2 Patientinnen der Anastrozolgruppe brachen wegen $\mathrm{Ne}-$ benwirkungen ab.
In einer aktuellen vergleichenden Analyse der beiden großen, kontrollierten Studien ATAC und BIG 1-98 unter Berücksichtigung unterschiedlicher Endpunkt-Definitionen kommen L.A. Vakaet et al., Brüssel, zu dem Ergebnis, dass Risikodifferenzen und relative Risikoreduktionen bei Letrozol (BIG 1-98) und Anastrozol (ATAC) für die untersuchten Endpunkte vergleichbar sind. Die Autoren planen eine formale, auf individuellen Patientendaten basierende Metaanalyse unter Berücksichtigung spezifischer Patientensubgruppen. Eine verlässliche Antwort auf die Frage, ob Letrozol und Anastrozol vergleichbare Wirksamkeit und Verträglichkeit zeigen, verspricht die derzeit durchgeführte Studie FACE (Femara vs Anastrozole Clinical Evaluation). Erste Ergebnisse werden in San Antonio 2007 erwartet.

\section{Östrogenrezeptor-Antagonist als Therapieop-} tion bei fortgeschrittenem Mammakarzinom

In der adjuvanten Therapie Hormonrezeptor(HR)-positiver Mammakarzinome und bei fortgeschrittener Erkrankung auch in der Firstline-Therapie werden bei postmenopausalen Patientinnen immer häufiger Nichtsteroidale Aromataseinhibitoren (NSAI) der dritten Generation, das heißt Letrozol oder Anastrozol, eingesetzt. Patientinnen, die unter dieser Behandlung ein Rezidiv oder eine Progression ihrer Tumorerkrankung erleiden, scheinen entsprechend den Hinweisen aus Phase-II-Studien von einer Weiterbehandlung mit dem steroidalen Aromatasehemmer Exemestan oder mit dem Estrogenrezeptor-Antagonisten Fulvestrant zu profitieren. In EFECT, einer ersten klinischen Studie der Phase III, wurden nun diese beiden Substanzen bei Patientinnen mit Progress unter NSAI miteinander verglichen. Insgesamt 693 Frauen wurden für die Studie rekrutiert. Die mediane Zeit bis zur Progression betrug in beiden Behand- lungsarmen 3,7 Monate. Die Zeit bis zum Progress, die objektive Responserate, und der Anteil der Patienten, die durch die Therapie einen klinischen Nutzen erfuhren, waren in beiden Behandlungsarmen praktisch gleich. Lediglich in der Dauer des Ansprechens ergab sich für Fulvestrant gegenüber Exemestan ein Vorteil von fast 4 Monaten (13,5 vs 9,8 Monate), der jedoch keine statistische Signifikanz erreichte. William Gradishar aus Chicago, der die Ergebnisse der Studie in San Antonio vorstellte, wies zusammenfassend darauf hin, dass Fulvestrant sich bei dieser Patientinnengruppe als vergleichbar wirksam und verträglich erwies wie Exemestan [3]. Ein zentraler Kritikpunkt an der Studie ist, dass Patientinnen mit Human Epidermal Growth Factor Rezeptor 2(HER2)-positivem Tumor nicht gezielt identifiziert und - entsprechend aktuellem Therapiestandard - mit dem monoklonalen anti-HER2-Antikörper Trastuzumab behandelt wurden.

\section{Referenzen}

1 Geisler J, et al: Letrozole tablets suppress tissue and plasma estradiol (E2), estrone and estrone sulfate levels more effectively than anastrozole in postmenopausal women with breast cancer. San Antonio Breast Cancer Symposium 2006; \#103.

2 Dixon JM, et al: Anastrozole and letrozole tablets in an investigation and comparison of quality of life, tolerability and morbidity (ALIQUOT). San Antonio Breast Cancer Symposium 2006; \#105.

3 Gradishar W, et al: Fulvestrant versus exemestane following prior non-steroidal aromatase inhibitor therapy: first results from EFECT, a randomized, phase III tria in postmenopausal women with advanced breast cancer. San Antonio Breast Cancer Symposium 2006; \#12.

Weitere Informationen bei

Novartis Pharma GmbH

Dr. Irene Roth

Leitung Kommunikation Onkologie

Tel. +49 911273 12-643, Fax -841

irene.roth@novartis.com

\title{
PharmaTicker+++ PharmaTicker+++ PharmaTicker+++ PharmaTicker+++
}

Sanofi-Aventis Deutschland GmbH. Im Dezember 2006 wurden die Ergebnisse der zweiten Interimsanalyse der Phase-III-Brustkrebsstudie BCIRG-006 bekannt gegeben. Die Daten zur Wirksamkeit und Sicherheit nach median 3-jährigem Follow-up bestätigen, dass die Behandlung mit Taxotere ${ }^{\circledR}$-haltigen Regimes in Kombination mit Herceptin ${ }^{\circledR}$ (Trastuzumab) bei Frauen mit HER2-positivem Brustkrebs im Frühstadium das erkrankungsfreie Intervall signifikant verlängert.

Sanofi-Aventis Deutschland GmbH

Cristina lannazzo

Tel. +49 69 305-24417, Fax -18802

cristina.iannazzo@sanofi-aventis.com
AstraZeneca GmbH. Auf dem 29. San Antonio Breast Cancer Symposium im Dezember 2006 wurden neue Daten vorgestellt, die das positive Nutzen/Risiko-Profil von Anastrozol (Arimidex) untermauern. Eine weitere Auswertung der ATAC-Studie (Arimidex Tamoxifen alone or in Combination) zeigte, dass unter Anastrozol signifikant weniger thromboembolische Ereignisse auftraten als unter Tamoxifen.

Weitere Informationen bei

Astra Zeneca GmbH

Frau Dagmar Hinz

Tel. +49 4103 708-3760

dagmar.hinz@astrazeneca.com
Roche Pharma AG. Das Komitee für Produkte der Humanmedizin der Europäischen Union (Committee for Human Medicinal Products/ CHMP) hat im Januar die Zulassungsempfehlung für das oral applizierbare Krebsmedikament Erlotinib $\left(\operatorname{Tarceva}^{\circledR}\right)$ in der Therapie des metastasierten Pankreaskarzinoms ausgesprochen.

Weitere Informationen bei

Roche Pharma AG

Herrn Dr. H.-U. Jelitto

Tel. +49 7624 14-2400, Fax -3366

\section{KARGER}




\section{Fortgeschrittenes kolorektales Karzinom}

Internationale Phase-III-Studie: Therapie mit Xeloda ${ }^{\circledR}$ zögert Fortschreiten der Erkrankung hinaus

Die Kombinationschemotherapie XELOX (orales Fluoropyrimidin Capecitabin (Xeloda ${ }^{\circledR}$ ) mit Oxaliplatin) zögert das Fortschreiten der Erkrankung ebenso wirksam hinaus wie FOLFOX4 (i.v. 5-FU/FS plus Oxaliplatin). Dies zeigt eine groß angelegte internationale PhaseIII-Studie (NO16967) bei Patienten mit fortgeschrittenem kolorektalen Karzinom, die bereits eine Chemotherapie erhalten hatten. Die viel versprechenden Ergebnisse deuten darauf hin, dass XELOX als neue wirksame Behandlungsoption in der Therapie des fortgeschrittenen kolorektalen Karzinoms eingesetzt werden kann.
Aufgrund der positiven Studienergebnisse beabsichtigt Roche weltweit Zulassungsanträge einzureichen.

Der Vorteil einer Behandlung mit Xeloda ist neben seiner wirksamen und sicheren Anwendung auch seine einfache orale Einnahme. Dies erspart den Patienten zahlreiche Arztbesuche oder Krankenhausaufenthalte, die durch die i.v.Gabe notwendig wären.

An der Phase-III-Studie nahmen 627 Patienten mit kolorektalem Karzinom aus 15 Ländern weltweit teil. Eingeschlossen waren Patienten, die bereits eine Chemotherapie erhalten hatten und deren Erkrankung danach entweder wieder aufgetreten oder weiter fortgeschritten war. Das primäre Studienziel bestand darin zu zeigen, dass die XELOX-Kombination ebenso wirksam das Fortschreiten der Krankheit oder den Tod hinauszögert wie FOLFOX4
Mace Rothenberg, Professor am Vanderbilt-Ingram Cancer Center und Leiter der Studie, erklärte: «Mit dem Nachweis, dass XELOX ebenso wirksam war wie FOLFOX4, liefert diese Studie den bisher stärksten Beleg dafür, dass Xeloda die intravenöse 5-FU-Chemotherapie bei der Behandlung von Patienten mit fortgeschrittenem koloraktalen Karzinom ersetzen kann.»

\section{Weitere Informationen bei}

Roche Pharma AG

Herrn Dr. H.-U. Jelitto

Tel. +49 7624 14-2400, Fax -3366

\section{Phase-III-Studie mit Erbitux: Pri- märer Endpunkt bei der First-line- Therapie von $\mathrm{mCRC}$ erreicht}

Die Merck KGaA hat bekannt gegeben, dass eine Phase-III-Studie zur Anwendung von Erbitux ${ }^{\circledR}$ (Cetuximab) plus Irinotecan-basierter Therapie den primären Endpunkt - Verlängerung der progressionsfreien Überlebenszeit bei Patienten mit zuvor unbehandeltem metastasierendem Kolorektalkarzinom (mCRC) - erreicht hat. Die als CRYSTAL (Cetuximab combined with iRinotecan in first line therapY for metaSTatic colorectAL cancer) bekannte internationale, randomisierte Phase-III-Studie untersuchte Patienten, die mit Erbitux + FOLFIRI (Irinotecan-basierte Chemotherapie) behandelt wurden, im Vergleich zu Patienten, die FOLFIRI erhielten.
«Wir sind über diese Ergebnisse sehr erfreut. Diese Daten demonstrieren eine Verbesserung der progressionsfreien Überlebenszeit bei der Erst-Linien-Behandlung mit einem gegen den EGFR gerichteten monoklonalen Antikörper im Rahmen einer kontrollierten klinischen Studie», erklärte Dr. Wolfgang Wein, Senior Vice President, Global Oncology Commercialization bei Merck. «Außerdem belegen diese Ergebnisse den Nutzen einer Erbitux-Verabreichung zusätzlich zur Chemotherapie als Erstlinien-Behandlung.»

Zum Nachweis eines Unterschieds bei der progressionsfreien Überlebenszeit für den Erbitux plus FOLFIRI-Arm gegenüber dem FOLFIRIArm wurden mehr als 1000 Patienten (Lang I, et al. ASCO 2006, abstract No. 3555) weltweit in die CRYSTAL-Studie eingeschlossen. Die
Ergebnisse sind zur Präsentation im Juni auf dem 2007 American Society of Clinical Oncology Annual Meeting in Chicago eingereicht.

Weitere Informationen bei

IntraMedic $\mathrm{GmbH}$

Frau Yvonne Söltl

Dornhofstr. 34

63263 Neu-Isenburg

Tel. +49 6102 7993-345, Fax -301

Yvonne_Soeltl@de.yr.com

\section{PharmaTicker+++ PharmaTicker+++ PharmaTicker+++ PharmaTicker+++}

Fresenius Biotech GmbH. Bei der Behandlung von Krebspatienten mit symptomatischem malignen Aszites erwies sich der trifunktionale Antikörper catumaxomab als wirksam und verträglich. Dies zeigen jetzt erstmals vorgestellten Phase-II/III-Daten mit 129 Patientinnen mit Ovarialkarzinom, die einer therapeutischen Punktion der Bauchhöhle bedurften.

Weitere Informationen bei

Fresenius Biotech $\mathrm{GmbH}$

Konzern-Kommunikation

Dr. Bernd Ebeling

Tel. +49 6172 608-2378, Fax -2294

pr-fre@fresenius.de
Wyeth Pharma GmbH. Für hervorragende Leistungen im Bereich der Forschung und Entwicklung wurde Wyeth Pharmaceuticals mit dem «Management of the Year» Scrip Award ausgezeichnet. Eine Jury wählte das Wyeth Forschungs- und Entwicklungsteam aus einer Vielzahl Arzneimittelhersteller aus. Die Zeitschrift Scrip World Pharmaceutical News zählt zu den wichtigsten Veröffentlichungen in der pharmazeutischen Industrie.

Wyeth Pharma

Pressestelle Anika Wichert

Tel. +49 251 204-2030, Fax -2033

WicherA@wyeth.com
Ortho Biotech Division of Janssen-Cilag GmbH. Der Proteasom-Inhibitor Velcade hat im Dezember 2006 die FDA-Zulassung für die Rezidivtherapie des Mantelzell-Lymphoms erhalten. Damit darf der Wirkstoff nun in den USA sowohl bei Patienten mit diesem aggressiven Subtyp des Non-Hodgkin-Lymphoms als auch bei Erkrankten mit multiplem Myelom nach lediglich einer Vorbehandlung eingesetzt werden.

Weitere Informationen bei

Janssen-Cilag GmbH

Kerstin Aschoff

Tel +49 2137 955-412

kaschoff@jacde.jnj.com 